\title{
Design of a Novel Dual - Band Planar Inverted F - Antenna for Mobile Radio Applications
}

\author{
N. Chattoraj ${ }^{1,}$, Qurratulain ${ }^{1,}$,

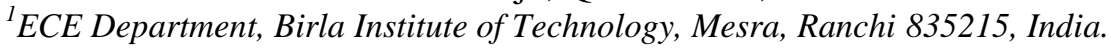 \\ nila.chwdhry@gmail.com,annie13ahmad@gmail.com. \\ J. Saxena ${ }^{1}$, S. Agarwal ${ }^{1}$, and K. V. Singh ${ }^{1}$ \\ ${ }^{I}$ ECE Department, Birla Institute of Technology, Mesra, Ranchi 835215, India. \\ saxena.juhi10@gmail.com, shikha87@gmail.com,vinyas_singh@yahoo.co.in.
}

\begin{abstract}
The design of a novel compact dual band PIFA (Planar Inverted F-Antenna) as an embedded antenna is reported in this paper for mobile radio applications. The new shorted planar antenna is realized using copper sheet of thickness $1 \mathrm{~mm}$ and air as the dielectric substrate. The radiating patch consists of a branch line slit to reduce the size of the antenna having a ground size of $\mathbf{3 6}$ $x 80 \mathrm{~mm}^{2}$. It can be used in internal mobile antennas for applications in GSM band, GCV (1.85 - 1.99GHz), PCS - 1900, IMT-2000 and UMTS 2100 networks. The simulations are done using the Ansoft High Frequency Structure Simulator (HFSS) software tool. The two operating frequency ranges obtained have good matching and radiation characteristics. The simulated results are well supported by measured results of the prototype of the antenna fabricated.
\end{abstract}

Index Terms - Antennas, HFSS, Impedance Bandwidth, PIFA, Planar Antennas.

\section{INTRODUCTION}

In recent years, there is a new trend in antenna development towards integrated antennas for mobile terminals that are created by embedding a small antenna into the mobile radio dielectric casing. This kind of concealed antenna is attractive for ergonomic reasons and is less likely to be damaged, compared with the conventional whip or rod antennas. For these reasons, they are becoming very popular for use in portable phone units. Furthermore, dual-band and tri-band mobile phones have gained popularity because of the multiple frequency bands used for wireless applications. Thus, the low profile of a PIFA makes it attractive for consideration as a concealed antenna[1], [2].

A number of new designs based on the Planar Inverted-F Antenna (PIFA) have become popular for handheld wireless devices because of their many attributes such as simple design ,lightweight, low cost, low profile, conformable nature and reliable performance [2]-[10].

In this paper we propose a new design for the Planar IFA using air as the dielectric substrate. Basic components of the PIFA include meandered rectangular planar copper radiating element of $1 \mathrm{~mm}$ thickness, ground plane, feed plate and shorting plate. The meandering in the patch leads to a large 
reduction in the required dimensions of the shorted patch for 900/1800/2100 band operation. The simulated and measured results have been compared and in the process a novel antenna is realized and fabricated.

\section{ANTENNA CONFIGURATION}

The 3D model of the proposed dual frequency planar inverted - $\mathrm{F}$ antenna as simulated in HFSS is shown in fig. 1(a) \& (b) .The proposed antenna is designed by cutting slits in a rectangular copper metal strip of size $16 \times 36 \mathrm{~mm}^{2}$ used as the conducting patch as shown in fig. 1(a). It also consists of a rectangular copper strip of size $36 \times 80 \mathrm{~mm}^{2}$ as the ground plane placed at a distance of $8 \mathrm{~mm}$ from the conducting patch as seen in fig. 1(b). The air acts as the dielectric substrate with a permittivity of 1 and substrate height of $8 \mathrm{~mm}$. The shorting plate of dimensions $3 \times 8 \mathrm{~mm}^{2}$ is used along with meander-type radiation patch in order to minimize the size of the antenna. The meandering in the patch leads to a large reduction in the required dimensions of the shorted patch for 900/1800/2100 $\mathrm{MHz}$ band operations.

The isometric view of the antenna designed in Auto-CAD is shown in fig. 2 and all the important dimensions are mentioned in Table I. The branch line slit consists of a main slit and a long folded branch slit as shown in fig. 2. The size of the top plate along with the location of the feed point with respect to the short circuit plate determine the resonant frequency and the overall bandwidth of the PIFA.

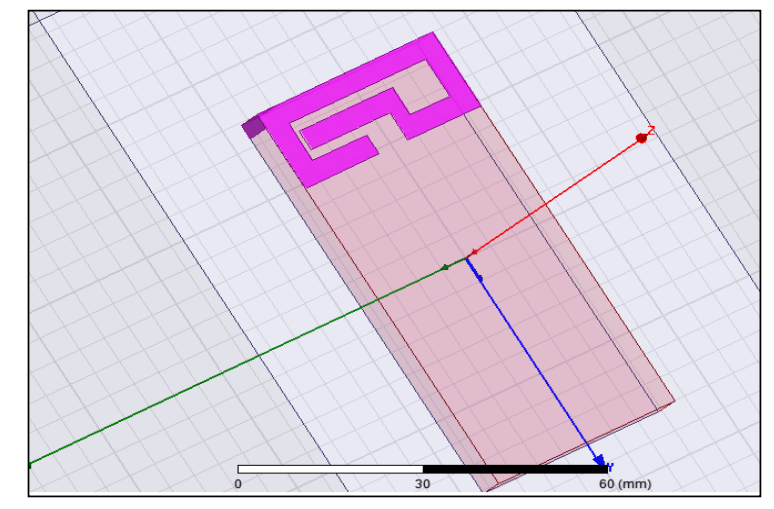

(a)

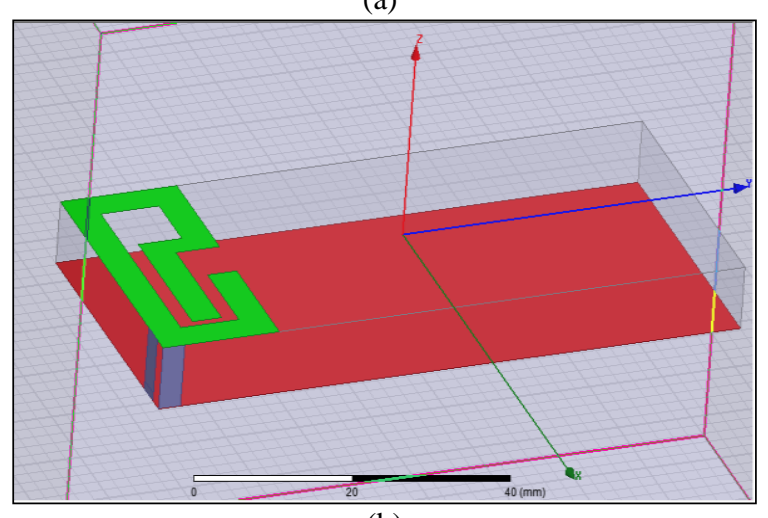

(b)

Fig 1. 3D model of the proposed dual band PIFA. 
TABLE I. DETAILED PARAMETERS OF THE PROPOSED PIFA

\begin{tabular}{ll}
\hline \multicolumn{1}{c}{ Parameters } & Dimension (mm) \\
\hline Size of the antenna radiator & $16 \times 36 \mathrm{~mm}^{2}$ \\
Size of the ground plane & $36 \times 80 \mathrm{~mm}^{2}$ \\
Size of feed plate & $3 \times 8 \mathrm{~mm}^{2}$ \\
Size of shorting plate & $3 \times 8 \mathrm{~mm}^{2}$ \\
Air gap or dielectric thickness & $8 \mathrm{~mm}$ \\
\hline
\end{tabular}

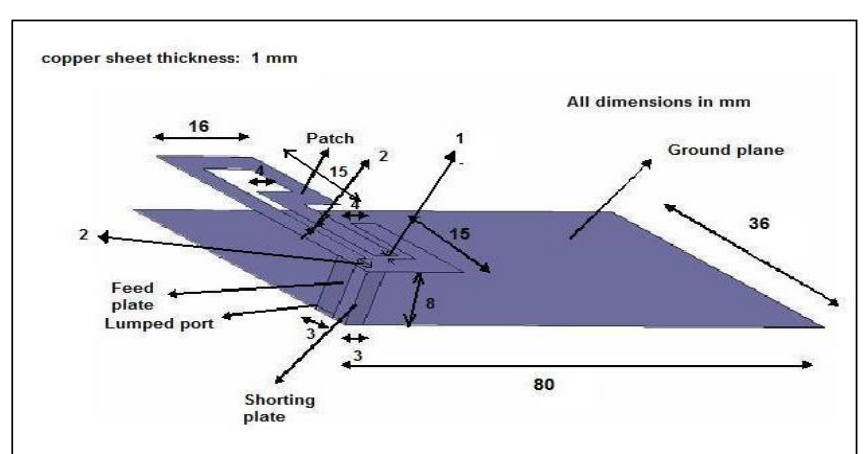

Fig 2. Isometric view of the proposed antenna.

The centre frequencies considered for designing of dual band PIFA in HFSS simulation software are $0.98 \mathrm{GHz}$ and $1.98 \mathrm{GHz}$. The simulation results are shown in the following section. Thus this antenna covers the Standards GSM(Global system of mobile communication), PCS(Personal communication systems), GCV(Ground Control Vehicle) and UMTS (Universal Mobile Telecommunication Services) applications. A list of the services are shown in Table II.

TABLE II. MOBILE APPLICATIONS WITH FREQUENCY BAND OF OPERATION

\begin{tabular}{ll}
\hline Wireless Applications & Bands of operation(GHz) \\
\hline GSM & $0.890-0.960 \mathrm{GHz}$ \\
GCV & $1.85-1.99 \mathrm{GHz}$ \\
PCS 1900 & $1.85-1.9 \mathrm{GHz}$ \\
UMTS 2100 & $1.9-2.17 \mathrm{GHz}$ \\
Bluetooth & $2.4-2.45 \mathrm{GHz}$ \\
\hline
\end{tabular}

\section{RESULTS AND DISCUSSION}

Simulations have been carried out with the Ansoft HFSS to determine the dual band PIFA's performance parameters of impedance bandwidth (VSWR $<2$ ), radiation patterns, peak gains and current distribution. The dual-band PIFA having the meander structure as shown in fig 2 was fabricated on the basis of the simulation results. Fig. 3 shows the structure of the manufactured antenna. It is clearly seen that the ground and radiator are cut out from copper plates and are connected through a SMA connector. The size of the feed plate is taken to be $3 \times 8 \mathrm{~mm}^{2}$. The soldering in the fabricated antenna is done by connecting the top plate or patch with the feed plate which is then soldered to the inner conductor or probe of the SMA connector. 

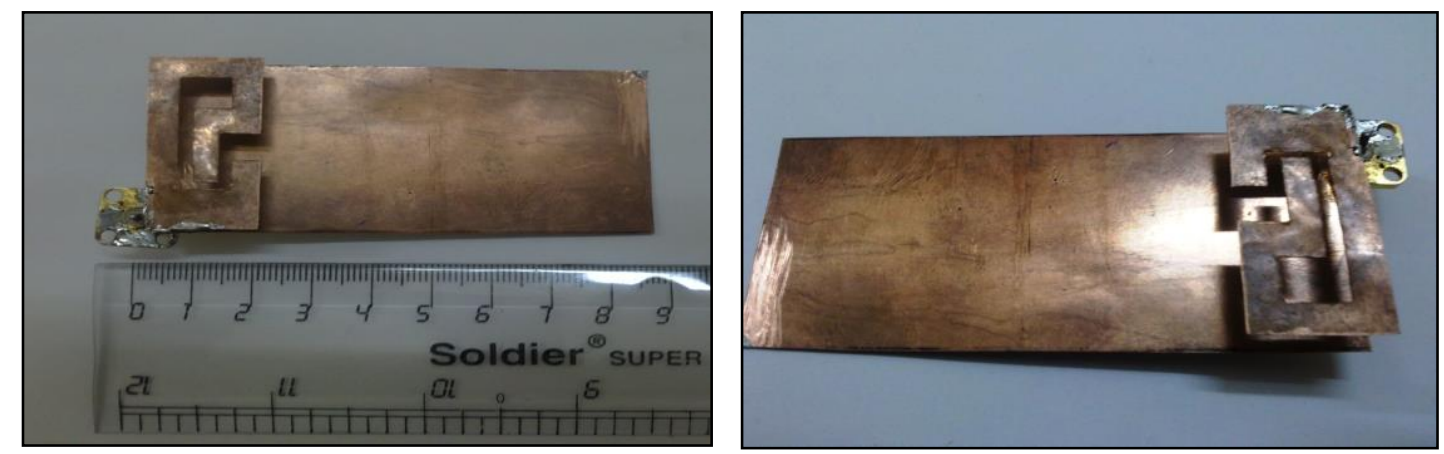

Fig 3. Snapshot of fabricated dual band PIFA.

The measurements were done using vector network analyzer (VNA, PNA N5230A, Agilent Technologies) as shown in fig. 4.

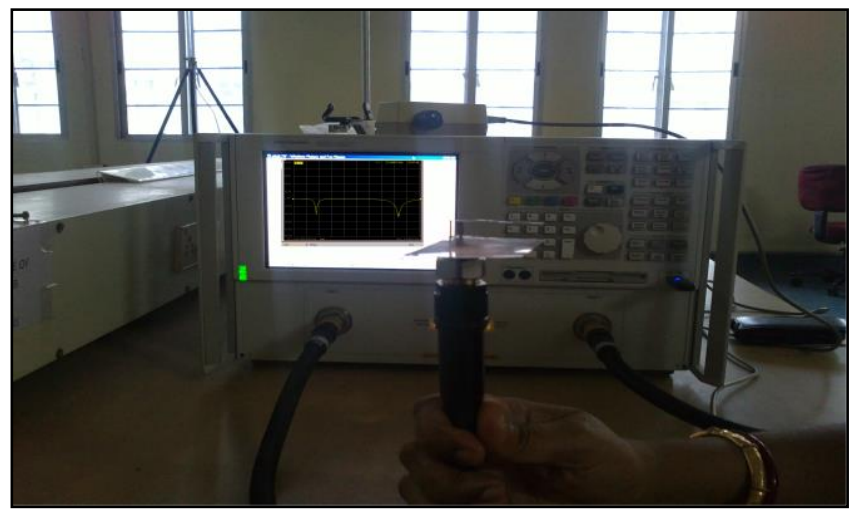

Fig 4. Photograph of the measuring instrument VNA.

\section{A. Impedance Bandwidth and Smith Chart}

In the comparison of simulated and measured results, $-10 \mathrm{~dB}$ bandwidth of the proposed dual-band PIFA has dual resonance characteristic. The comparison of the return loss curves for simulated and measured readings are plotted in fig. 5(a). We can clearly see that the two frequencies of operation are $0.98 \mathrm{GHz}$ and $1.98 \mathrm{GHz}$ for simulated return loss curve as shown in fig. 5(a). The plot dips at these two values of frequencies indicating that the $S_{11}$ values are lowest at these frequencies and that this antenna will reflect the least amount of power back toward the input driving port. Hence, these are the operating frequencies. We observe that the bandwidth at operating frequency $0.98 \mathrm{GHz}$ is found out to be $300 \mathrm{MHz}$ while that at $1.98 \mathrm{GHz}$ its value increases to $800 \mathrm{MHz}$ in simulation. These are sufficient values for efficient operation of antenna.

The S-parameter plot obtained from the fabricated antenna has the same shape as seen in the simulated results. However, the operating centre frequencies are found to be slightly varying from the simulation results as seen in fig. 5(a) \& (b). The reasons for this variation may be due to losses occurred during fabrication which may consist of effects of soldering. The simulation is done under ideal conditions in HFSS software and the atmospheric factors (no anechoic chamber used) are not considered .The RF cable used for measurements can also lead to losses and thus variation of the 
measured result from the simulated result. The bandwidth of the higher frequency peak is found to be greater than that of the lower peak as seen in the return loss curves.

The simulated and measured values of VSWR lie between 1 and 2 as shown in fig. 5(b), which shows characteristics of a good antenna. Also the VSWR of the second peak shows that the frequency range of operation at centre frequency of $1.98 \mathrm{GHz}$ is more as observed in the simulation results and supported by measured results .

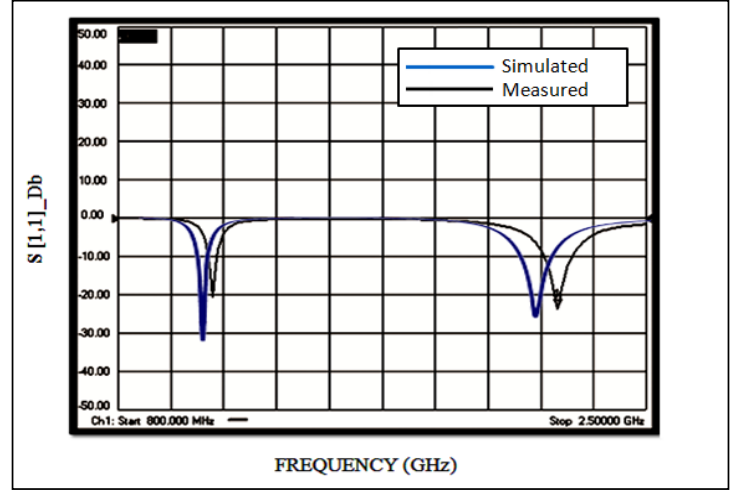

(a)

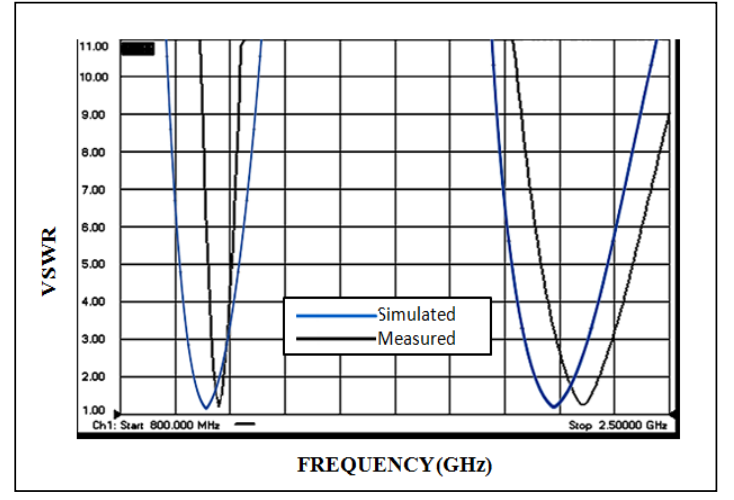

(b)

Fig 5. Comparison of Simulated and measured results for the proposed PIFA (a) Return loss curve (b) VSWR curve.

The comparison of the simulated and measured results at the two resonant frequencies is shown in Table III.

TABLE III. COMPARISON OF THE SIMULATED AND MEASURED RESULTS

\begin{tabular}{lcccc}
\hline Parameters considered & \multicolumn{2}{c}{ For First Resonance obtained } & $\begin{array}{c}\text { For Second Resonance } \\
\text { obtained }\end{array}$ \\
\cline { 2 - 5 } & \multicolumn{2}{c}{$\begin{array}{c}\text { Simulation results Measured } \\
\text { Results }\end{array}$} & $\begin{array}{c}\text { Simulation results Measured } \\
\text { Results }\end{array}$ \\
\hline Centre frequency of this & $0.98 \mathrm{GHz}$ & $1.103 \mathrm{GHz}$ & $1.98 \mathrm{GHz}$ & $2.225 \mathrm{GHz}$ \\
band & & & & \\
Bandwidth & $300 \mathrm{MHz}$ & $200 \mathrm{MHz}$ & $800 \mathrm{MHz}$ & $700 \mathrm{MHz}$ \\
VSWR & 1.08 & 1.285 & 1.14 & 1.354 \\
Impedance matching & $50 \Omega$ & $48.23 \Omega$ & $50 \Omega$ & $48.99 \Omega$ \\
\hline
\end{tabular}

The matching at the two operating centre frequencies of the dual band PIFA is shown in fig. 6(a) \& (b). The measured resistance for the coaxially fed antenna at the first resonant frequency of $1.07 \mathrm{GHz}$ is $48.23 \Omega$ and at the second resonant frequency of $2.2 \mathrm{GHz}$ is $48.99 \Omega$. The matching is considered to be at $50 \Omega$ for the lumped port used for excitation. 


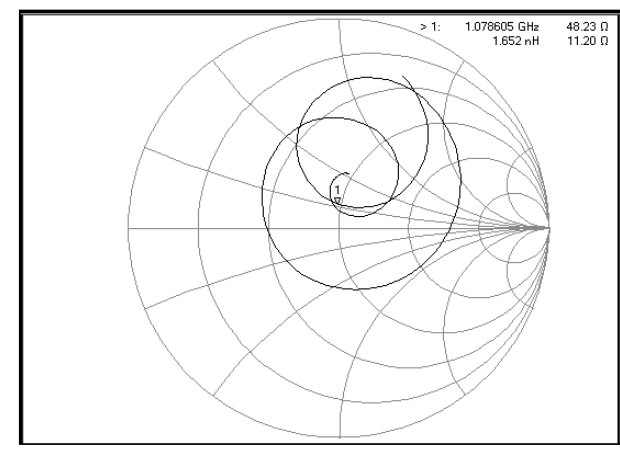

(a)

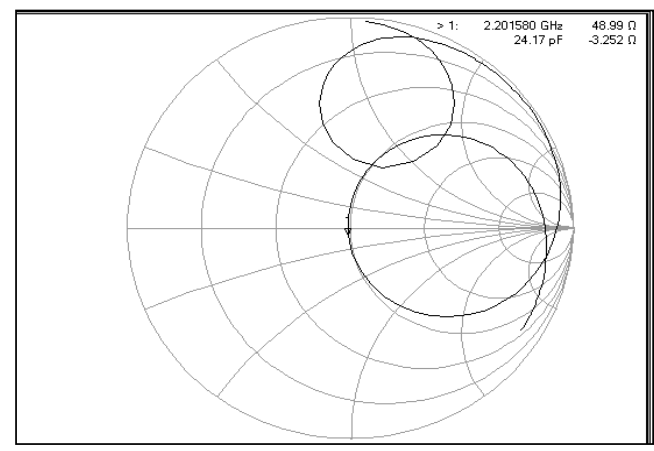

(b)

Fig 6. Measured smith chart of the dual band PIFA at the operating frequencies (a) At $0.98 \mathrm{GHz}$ (b) At $1.98 \mathrm{GHz}$.

\section{B. Radiation Patterns}

The simulated radiation patterns at the two operating frequencies are as shown in fig. 7(a) \& (b). It is clearly seen that the radiation patterns of E-plane are monopole like and H-plane radiation patterns show almost omni-directional characteristics.

The radiation pattern at lower frequency is showing omni-directional nature as shown by the simulated 3D radiation pattern in fig. 8(a). The value of maximum gain is obtained from the simulated radiation pattern. The value of maximum gain obtained is more than 2 at this frequency which is quite decent. The simulated maximum gain is $2.154 \mathrm{~dB}$ for $0.98 \mathrm{GHz}$ and $4.646 \mathrm{~dB}$ for $1.98 \mathrm{GHz}$. However, the radiation pattern characteristic at the higher frequency of operation $(1.98 \mathrm{GHz})$ is more directional than that at $0.98 \mathrm{GHz}$ as shown in fig. 7(b) \& 8(b). As a result the maximum gain at this frequency is quite high (4.646) due to the directionality obtained at this frequency of operation.

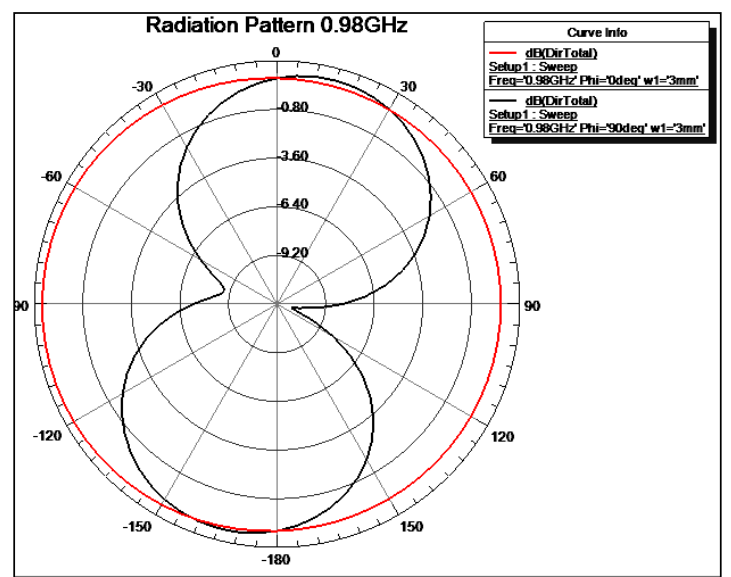

(a) At $0.98 \mathrm{GHz}$

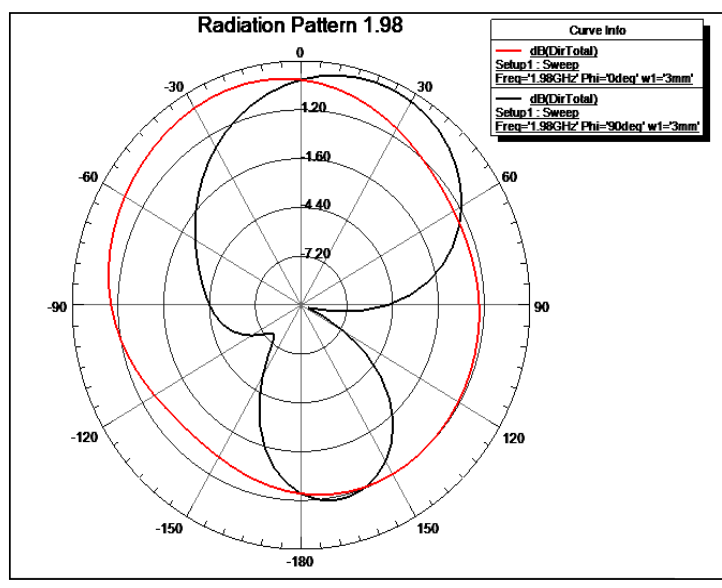

(b)At $1.98 \mathrm{GHz}$

Fig. 7. Simulated radiation patterns for the proposed PIFA at the dual resonances 


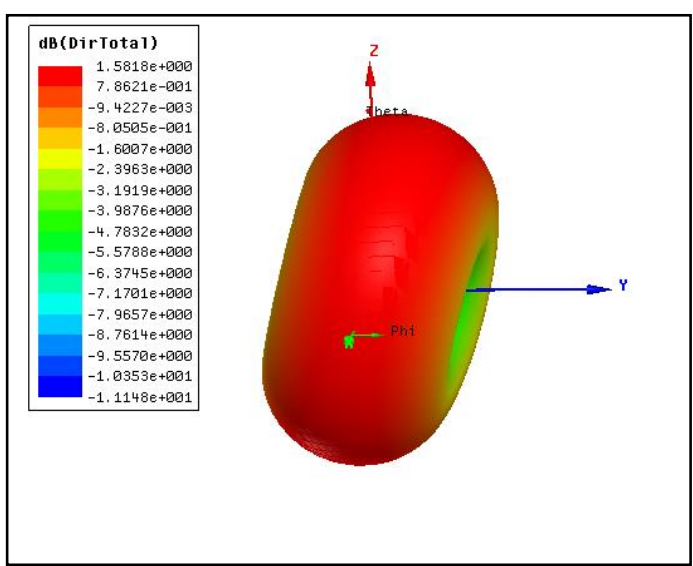

(a) At $0.98 \mathrm{GHz}$

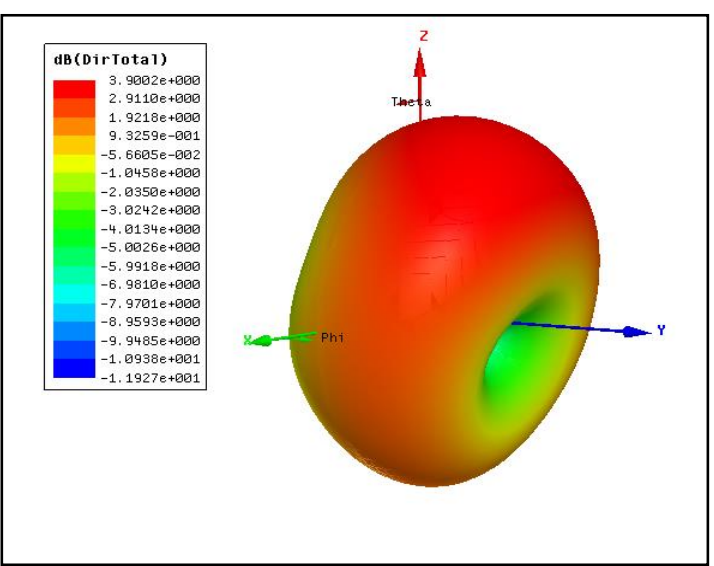

(b)At $1.98 \mathrm{GHz}$

Fig 8. Simulated 3D radiation patterns for the proposed PIFA.

\section{Current Distribution}

PIFA has very large current flow on the under surface of the planar element and the ground plane compared to the field on the upper surface of the element. Due to this behaviour PIFA is one of the best candidates when talking about the influence of external objects that affect the antenna characteristics. The surface current distribution of the proposed dual band PIFA at the two resonant frequencies is shown in fig. 9(a) \& (b). The maximum current distribution is close to the short pin and decreases away from it.

The main slit has an open end at the patch boundary, and the long folded branch slit in the shorted patch are for effectively meandering the excited patch surface currents. The slits meander the excited patch currents in the shorted patch.
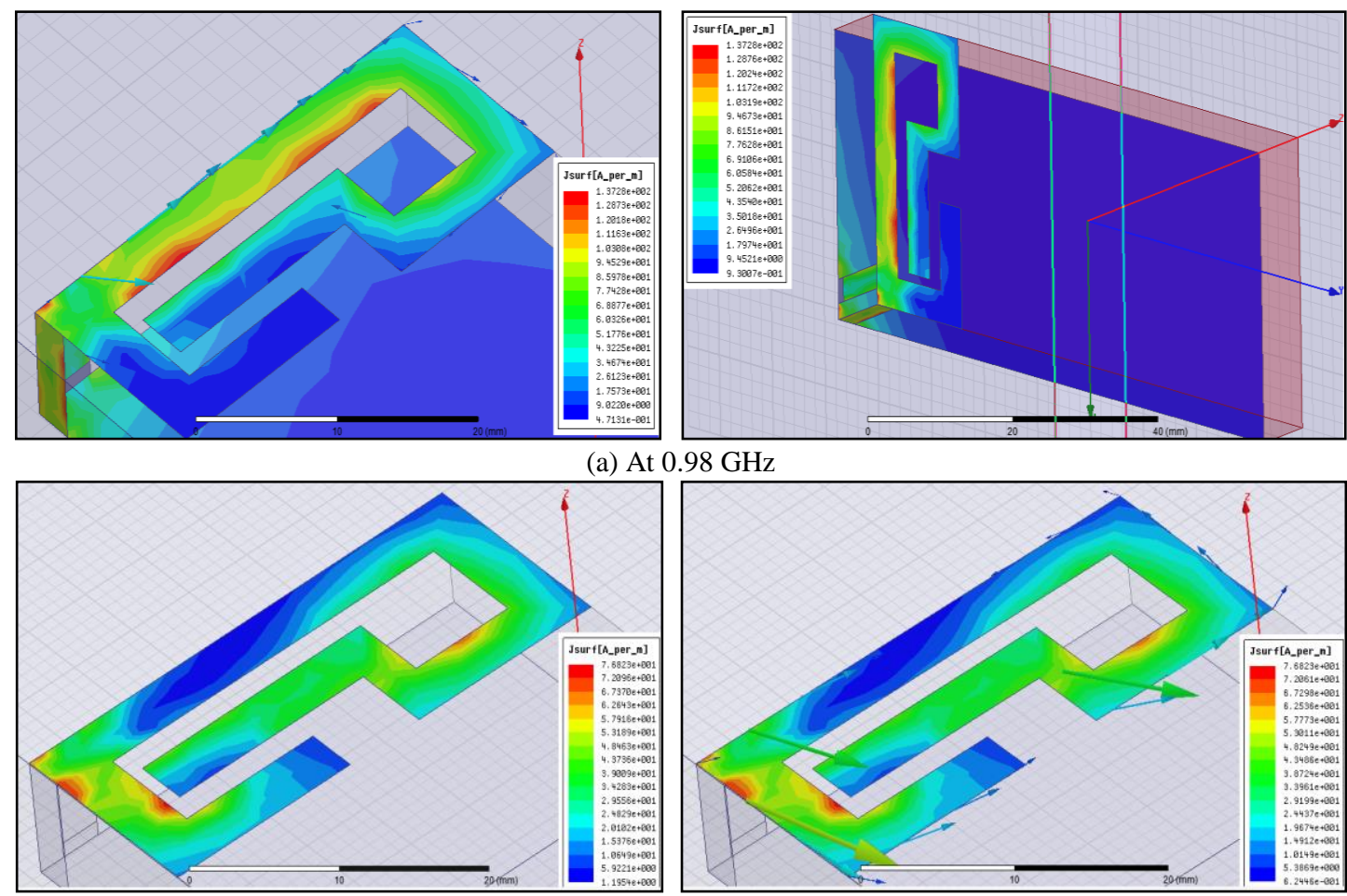

(b) At $1.98 \mathrm{GHz}$

Fig. 9. Surface current distribution for the proposed dual band PIFA (a) At $0.98 \mathrm{GHz}$ (b) At $1.98 \mathrm{GHz}$ 


\section{CONCLUSION}

In this paper a dual band PIFA is designed, simulated ,fabricated and tested successfully. The designed PIFA is a compact antenna. The gain and bandwidth of the antenna is limited. Gain can be increased by increasing the length of the antenna. However, we need to consider the size constraint. The results obtained from the fabricated antenna depict the same behaviour as depicted by the simulated design. The fabricated antenna is hence a dual-band PIFA operating at centre frequencies of $1.103 \mathrm{GHZ}$ and $2.225 \mathrm{GHZ}$ with acceptable bandwidths and VSWR values at both the frequencies. The shift in the measured centre frequencies is because the measurement is done in uncontrolled environment due to unavailability of anechoic chamber. The radiation patterns and current distribution along with the measured smith chart plots are also presented. The variations in fabricated results from the simulated results are within tolerable limits. The variations in the fabricated results from the simulated results can be reduced by reducing the losses occurred during fabrication. From fabricated results it is seen that the antenna can be used for Bluetooth and ISM bands too.

\section{ACKNOWLEDGMENT}

The authors would like to acknowledge and thank the support of Birla Institute of Technology (Mesra) for providing the facility and equipment for this work.

\section{REFERENCES}

[1] D. M. Pozar: “Microwave Engineering” John Wiley \& Sons, 1998.

[2] R. Hossa, A. Byndas, and M. E. Bialkowski," Improvement of Compact Terminal Antenna Performance by Incorporating Open-End Slots in Ground Plane", IEEE Microwave And Wireless Components Letters, VOL. 14, NO. 6,pp. 283-285, June 2004.

[3] J-H Lu and K-L Wong, "Slot-loaded, meandered rectangular microstrip antenna with compact dual frequency operation", Electronic Letters ,Vol. 34 No. 11,pp. 1048-1050,28th May 1998.

[4] R. Chair, K.M. Luk, K.F. Lee, "Miniature Multilayer shorted patch antenna", Electronic Letters, Vol. 36,No. 1, pp. 34. 6th January, 2000.

[5] T-W Chiou and K-L Wong," Designs of Compact Microstrip Antennas with a Slotted Ground Plane", pp.732-735, IEEE 2001.

[6] Dr. S. Raghavan, N. Jayanthi, "Design Of Planar Inverted-F Antenna For Wireless Application", WSEAS TRANSACTIONS on COMMUNICATIONS, Issue 8, Volume 8, pp. 863-872, August 2009.

[7] H. T. Chattha, Y Huang, M. K. Ishfaq, S. J. Boyes ,'A Comprehensive Parametric Study of Planar Inverted-F Antenna', Wireless Engineering and Technology, Vol. 3, pp. 1-11, 2012.

[8] J-S Kuo and K-L Wong ,"Dual-Frequeny Operation Of A Planar Inverted-L Antenna With Tapered Patch Width", Microwave And Optical Technology Letters, Vol. 28, No. 2, pp. 126-127,January 20, 2001.

[9] K Chang, G-Y Chen, J-S Sun, and Y. D. Chen," PIFA Antenna with Coupling Effect for Bandwidth Enhanced Design and Measurement", PIERS Proceedings, Xi'an, China, pp. 1807 -1810, March 22-26, 2010.

[10] Sung-Keun Jeon, Nam Kim, Seung-Woo Lee, Sang-Myeong Park, and Byoung-Jun Jang," Design of Dual- band PIFA for WLAN", PIERS Proceedings, Hangzhou, China, pp. 212 -216, March 24-28, 2008. 